\title{
Woodchuck Hepatitis Virus Post-Transcriptional Regulation Element (WPRE) Promotes Anti-CD19 BiTE Expression in Expi293 Cells
}

\author{
Reza Moazzami ${ }^{1}$, Hasan Mirzahoseini ${ }^{1}$, Leila Nematollahi ${ }^{1}$, Farzaneh Barkhordari ${ }^{1}$, Mozhgan \\ Raigani $^{1}$, Fatemeh Hajari Taheri ${ }^{2}$, Fereidoun Mahboudi ${ }^{1}$ and Fatemeh Davami ${ }^{{ }^{*}}$ \\ ${ }^{1}$ Biotechnology Research Center, Pasteur Institute of Iran, Tehran, Iran; \\ ${ }^{2}$ Department of Immunology, Hybridoma Lab, Pasteur Institute of Iran, Tehran, Iran
}

Received 12 October 2020; accepted 22 December 2020; published online19 May 2021

\begin{abstract}
Background: Bispecific antibodies represent an important class of mAbs, with great therapeutic potentials due to their ability to target simultaneously two distinct epitopes. The generation of functional bispecific antibodies with the highest possible yields is particularly critical for the production of these compounds on industrial scales. Anti$\mathrm{CD} 3 \times \mathrm{CD} 19 \mathrm{bsAb}$ is a bispecific T-cell engager (BiTE) currently used for treating ALL. Herein, we have tried to optimize the expression level of this antibody in mammalian hosts. Methods: WPRE sequence was incorporated at the $3^{\prime}$ end of the expression cassette. This modification resulted in a notable about two-fold increase in the expression of the bsAb in the Expi293 cell line. Results \& Conclusion: Follow-up flow cytometry analysis demonstrated the binding properties of the produced antibody at acceptable levels, and in vitro bioactivity assays showed that this product is potent enough for targeting and destroying CD19-positive cells. Our findings show that WPRE enhances the expression of this type of bispecific mAbs in HEK-293 family cell lines. This approach can be used in biopharma industry for the mass production of anti-CD3 $\times$ CD19 bispecific antibody. DOI: 10.52547/ibj.25.4.275
\end{abstract}

Keywords: Acute lymphoblastic leukemia, Bispecific antibodies, Monoclonal antibody

Corresponding Author: Fatemeh Davami

Biotechnology Research Center, Pasteur Institute of Iran (IPI), No. 69, Pasteur Ave, Tehran, Iran; E-mail: f.davami@gmail.com

\section{INTRODUCTION}

I mmunotherapy of cancer is considered as a successful approach in treating a wide spectrum of malignancies, including leukemia and lymphoma ${ }^{[1]}$. A range of cancer immunotherapy agents have been introduced including mAbs, checkpoint inhibitors, and cancer vaccines. mAbs have a major impact on cancer immunotherapy, as shown by their successful implementation for more than a decade ${ }^{[2]}$. Presently, there is a broad range of mAbs capable of specifically targeting different cell surface antigens, making them a valuable and reliable tool in this new targeted therapy ${ }^{[3]}$. Despite their undeniable applications in research, diagnosis, and therapy, native mAbs suffer from some critical limitations viz a restricted activity, Fc-dependent mechanism of action, inadequate pharmacokinetic profile, and limited tissue accessibility ${ }^{[4-6]}$. To evade such limitations, numerous strategies, including engineered antibodies, have been developed in recent years ${ }^{[7]}$.

Bispecific antibodies are a successful subclass of engineered antibodies, which hold promise as a method for increasing $\mathrm{mAb}$ efficacy through the combination

\section{List of Abbreviations:}

ALL, acute lymphoblastic leukemia; B-ALL, B-cell precursor acute lymphoblastic leukemia; bsAb, bispecific antibody; CD, chemically defined; CHO, Chinese hamester ovary; DAB, 3,3'-diaminobenzidine; E/T, effector/target; FCS, fetal calf serum; GFP, green fluorescent protein; HEK, human embryonic kidney; mAb, monoclonal antibodies; PB, peripheral blood mononuclear cells; PercP, peridinin chlorophyll protein complex; PTRE, post-transcriptional regulatory elements; WPRE, woodchuck hepatitis virus posttranscriptional regulation 
of two activities in one molecule. These activities include neutralizing two ligands, inhibiting two receptors, crosslinking two receptors on one cell, and redirecting cytotoxic immune cells ${ }^{[8,9]}$. Until now, regulatory authorities have approved three bispecific antibodies, blinatumomab (Amgen, USA), catumaxomab (TriOn Pharma, UK) $)^{[10]}$ and Emicizumab (Roche, Switzerland), for clinical uses ${ }^{[11]}$. Blinatumomab is the first in the class member of the bispecific $\mathrm{T}$ cell-engaging (BiTE®) $\mathrm{mAbs}$, which can direct $T$ cells toward target tumor cells ${ }^{[12]}$. The simultaneous binding of blinatumomab to cytotoxic $\mathrm{CD}^{+} \mathrm{T}$ cells and $\mathrm{CD} 19^{+} \mathrm{B}$ cells results in malignant $\mathrm{B}$ cell lysis $^{[13]}$, while that of the CD19 marker and CD3 causes the $\mathrm{T}$-cell activation, leading to the upregulation of INF- $\gamma$, TNF- $\alpha, \mathrm{CD} 69, \mathrm{CD} 25$, and CD2, including $I L s-2,-6$, and -10 genes ${ }^{[14]}$. Additionally, the secretion of granzymes and perforin from cytotoxic $\mathrm{T}$ lymphocytes mediates cell lysis ${ }^{[15]}$, giving rise to polyclonal T-cell activation and proliferation ${ }^{[1]}$. CD19 is the first B-lineage-specific antigens appearing on the cell surface of B lymphocytes retained in all stages of B-cell development, except in plasma cells ${ }^{[16]}$. Blinatumomab is applied to treat relapsed or refractory Philadelphia chromosome-negative B-ALL ${ }^{[17]}$. ALL is a type of lymphoid line malignancies, marked by the rapid dispersion of lymphoblasts in bone marrow ${ }^{[18]}$. In spite of the great progress recorded in chemotherapy and hematopoietic stem cell transplantation, B-ALL patients continually experience the high rates of relapse, highlighting the need for novel therapies ${ }^{[19]}$.

Currently, a variety of bispecific antibodies have been successfully expressed in several hosts, and some of them are in trials. An anti-CD123 $\times$ anti-CD3 in BiTE-Fc format was transiently expressed in the $\mathrm{CHO}$ $\mathrm{K} 1$ cell line for treating leukemia ${ }^{20]}$. Moreover, there are promising results for treating $\mathrm{P}$-cadherin expressing solid tumors by the application of a bsAb targeting Pcadherin and CD3 in DART-Fc format in CHO cell ${ }^{[21]}$. Recently, Roche has developed a vascular endothelial growth factor-A and angiopoietin-2 targeting bispecific antibodies in CrossMab format for treating age-related macular degeneration. This product, expressed in the HEK293-F system (Invitrogen, USA), is in clinical trial Phase $\mathrm{II}^{[22]}$. Expi293FTM cells were used for the production of bispecific CrossMab that targets CD4 and the HIV envelope glycoprotein ${ }^{[23]}$. Expi293FTM cells (Gibco, USA) are a suspension derivative of the HEK cells, adapted to grow at high cell densities in a CD, serum-free medium for transient gene expression $^{[21]}$. In mammalian hosts, the transient gene expression, in contrast to the generation of stable cell lines, ensures the rapid production of enough target proteins for biophysical and biochemical assessment, as well as preclinical studies ${ }^{[24-26]}$. Engineering the expression vector, to enhance protein production levels per gene copy, is a prominent approach used in transient gene expression ${ }^{[27]}$. Importantly, PTREs in mammalian cells can improve gene expression levels through the increased mRNA stability, polyadenylation, translation, and export to cytoplasm $^{[27,28]}$. Existing evidence indicates that the presence of WPRE may result in increased expression levels of target proteins through acting on polyadenylation, mRNA export, or translation ${ }^{[28-30]}$. Therefore, WPRE can be used to enhance the expression levels of target proteins in transient gene expression. Curiously, some reports have demonstrated the ability of WPRE to elevate the expression level of transgenes in a variety of cell lines ${ }^{[27,31]}$.

To date, the impact of WPRE on transgene expression, which is cell line- and construct-dependent, has not yet been investigated in bispecific production and in Expi293 cells. Hence, we aimed at studying the effect of WPRE on the expression level of anti-CD3 $\times$ CD19 bsAb (blinatumomab) in the Expi293 cell line.

\section{MATERIALS AND METHODS}

\section{Cell lines and reagents}

Suspension-adapted Expi293F ${ }^{\mathrm{TM}}$ cells was purchased from Invitrogen and NALM-6 and Jurkat cell lines from the National Cell Bank of Iran (Tehran). CD, protein-free Expi293 ${ }^{\mathrm{TM}}$ Expression Medium, and ExpiFectamine ${ }^{\text {TM}} 293$ transfection Reagent were procured from Invitrogen. Penicillin/streptomycin and L-glutamine were also obtained from Invitrogen. NiNTA Superflow resin was acquired from Qiagen (USA). HRP-conjugated anti-polyhistidine antibody, trypan blue, and DAB were bought from SigmaAldrich (USA). Calcein AM viability kit and Saponin were from Trevigen (USA) and Roche (Mannheim, Germany), respectively. Cell separation media were purchased from Cedarlane (Canada), and FCS and RPMI 1640 from Gibco (Karlsruhe, Germany). An anti-polyhistidine PerCP-conjugated antibody was acquired from R\&D systems (Minnesota, USA).

\section{Cell culture conditions}

Expi293FTM cells were cultured in a serum-free and CD Expi293 ${ }^{\mathrm{TM}}$ Expression Medium supplemented with $2 \mathrm{mM}$ of penicillin/streptomycin. The cells in the culture media were grown in glass bottles on a shaker in a humidified 5-8\% $\quad \mathrm{CO}_{2}$ incubator at $125 \mathrm{rpm}$ at $37{ }^{\circ} \mathrm{C}$. Transfection was performed using ExpiFectamine ${ }^{\mathrm{TM}} 293$ reagent according to the manufacturer's instruction. Every three days, the cells were passaged at a density of $3 \times 10^{5}$ cells $/ \mathrm{mL}$, and the 
cell density and viability ratio were evaluated using trypan blue exclusion methodology.

\section{Expression cassettes}

To make the blinatumomab expression constructs, pcDNA3.1 (+) vector (from Invitrogen) was used as the backbone. The synthetic sequences corresponding to the bsAb and WPRE were obtained from a commercial supplier on a single $\mathrm{pGH}$ plasmid. For this study, two constructs were prepared: (1) pcDNABi (7 $\mathrm{kb})$ by inserting the bsAb sequence extracted from the pGH plasmid (using NheI and HindIII cut sites) into the destination vector pcDNA3.1, and (2) pcDNABiW $(7.5 \mathrm{~kb})$ by inserting "bsAb + WPRE" sequence extracted from the pGH plasmid (using NheI and EcoRI) into pcDNA3.1.

\section{Transfection of Expi293F cells}

Cells were transfected using cationic lipid-based ExpiFectamine ${ }^{\mathrm{TM}} 293$ Reagent, according to the manufacturer's protocol. One day before transfection, $2.5 \times 10^{6}$ cells $/ \mathrm{mL}$ were seeded onto a six-well plate, followed by the addition of $2 \mathrm{~mL}$ of Expi293 Expression Medium with no supplement. The next day, a transfection cocktail, formed at a ratio of $2.7 \mu \mathrm{g}: 1 \mu \mathrm{L}$ (DNA-ExpiFectamine ${ }^{\mathrm{TM}} 293$ Reagent), was diluted in $200 \mu \mathrm{L}$ of serum-free media. After 30 minutes of incubation at room temperature, DNA and ExpiFectamine ${ }^{\mathrm{TM}} 293$ were added to the cells. In the next step, i.e. 16-18 hours after transfection, $10 \mu \mathrm{L}$ of ExpiFectamine $^{\mathrm{TM}}$ Transfection Enhancer 1, and $100 \mu \mathrm{L}$ of ExpiFectamine ${ }^{\mathrm{TM}}$ Transfection Enhancer 2 were added to each well according to the manufacturer's protocol. The cells harvested on day seven were centrifuged $200 \times \mathrm{g}$ at room temperature for five minutes, and the supernatant fractions were kept at -20 ${ }^{\circ} \mathrm{C}$ for future analysis. To survey the effect of WPRE on $\mathrm{mAb}$ expression in Expi293F $\mathrm{F}^{\mathrm{TM}}$ cells, the GFPexpressing pb513b1 plasmid was used as an internal control vector for the normalization of sample-tosample transfection efficiency variations using flow cytometry assays after 48 and 72 hours following transfection. For flow cytometry, viable cells were gated and analyzed quantitatively using CyFlow software to evaluate the expression of GFP.

\section{Target and effector cell preparation}

CD19-positive B cell (NALM-6) and CD3-positive T cell (Jurkat) lines were cultured in 10\% FCS supplemented with complete RPMI 1640. Blood samples were obtained from healthy donor volunteers. Ficoll density gradient centrifugation was used for isolating PBMCs from the buffy coat. Erythrocyte lysis buffer was used for erythrocytes removal (15 minutes at room temperature), followed by centrifugation at $600 \times \mathrm{g}$ for 5 minutes. Lysed erythrocytes containing supernatant was discarded, and the cells were resuspended in PBS. Then the platelets were removed with an extra centrifugation step at $100 \times \mathrm{g}$ for 15 minutes. PBMCs were resuspended in RPMI 1640 to be used the following day without stimulation.

\section{Protein purification}

Antibody purification was performed by Ni-NTA superflow resin. Filtrated supernatant (using a $0.45-\mu \mathrm{m}$ filter) was introduced to a $1-\mathrm{mL}$ column at $1 \mathrm{~mL} / \mathrm{min}$ flow rate. Column equilibration was performed by applying an equilibration buffer containing $\mathrm{NaH}_{2} \mathrm{PO}_{4}$ (50 mM), $\mathrm{NaCl}(300 \mathrm{mM})$, and imidazole (10 mM), pH 8.0, and the sample was then loaded on the column. In the next step, a wash buffer, comprising of $\mathrm{NaH}_{2} \mathrm{PO}_{4}$ (50 $\mathrm{mM}), \mathrm{NaCl}(300 \mathrm{mM})$, and imidazole $(20 \mathrm{mM})$, $\mathrm{pH} 8.0$, was used, and in the end, the column was eluted by an elution buffer consisting of $\mathrm{NaH}_{2} \mathrm{PO}_{4}(50$ $\mathrm{mM}), \mathrm{NaCl}(300 \mathrm{mM})$, and imidazole (250 mM), $\mathrm{pH}$ 8.0. The bsAb containing fraction was collected in 1.5$\mathrm{mL}$ vials and kept at $-20{ }^{\circ} \mathrm{C}$. A Nanodrop spectrophotometer was used to measure the protein concentrations.

\section{SDS-PAGE analysis and Western blotting}

The expressed $\mathrm{mAb}$ was measured by SDS-PAGE and Western blotting. Purified fractions were subjected to $12 \%$ polyacrylamide gel (SDS-PAGE) and stained with Coomassie Brilliant Blue. For the Western blot analysis, separated proteins were transferred onto a nitrocellulose membrane using a semidry transfer cell (BioRad, USA). The membrane was blocked in $4 \%$ bovine serum albumin at $4{ }^{\circ} \mathrm{C}$ overnight, then incubated with HRP-conjugated anti-polyhistidine antibody (1:1000 dilution) for 120 minutes. The bands were then visualized by DAB.

\section{Flow cytometry analysis}

Binding properties of the expressed bispecific mAb was assessed by flow cytometry (Partec, Germany). For this purpose, $1 \times 10^{6}$ cells (Jurkat, PBMC, NAML6 , or $\mathrm{CHO})$ were mixed with bscCD19 $\times$ CD3 $(200$ $\mu \mathrm{g} / \mathrm{mL}$ in PBS $)$ at $4^{\circ} \mathrm{C}(50 \mu \mathrm{L}$ of final volume $)$ for 30 minutes. Subsequently, the cells were washed twice with FACS buffer (containing PBS and 1\% FCS) and centrifuged at $400 \times \mathrm{g}$ at $4{ }^{\circ} \mathrm{C}$ for 5 minutes. Cells were then incubated with secondary antibody (anti-His-tag PercP-conjugated antibody) on ice in the dark for 30 minutes, washed twice in the same conditions and analyzed by flow cytometry in the FL3 channel. The experiment was negatively controlled using the His-tag antibody. Flow cytometry output was analyzed by CyFlow (Partec, Germany). 


\section{Bioactivity assay}

The bsAb cytotoxicity was analyzed using the cytotoxic assay. PBMC preparation was performed as explained above, and T-cell isolation was performed using nylon wool method ${ }^{[32]}$. About $1.2 \times 10^{7}$ NALM-6 cells were labeled, in a cell culture medium, with 10 $\mu \mathrm{M}$ of calcein-AM (Tervigen, USA) at $37^{\circ} \mathrm{C}$ for 30 minutes. After washing two times in the cell culture medium, the cells were adjusted to $3 \times 10^{5}$ cells $/ \mathrm{ml}$ density in RPMI (10\% FCS), and about 30,000 cells were aliquoted in $100-\mu 1$ volumes for application per reaction assay. Isolated PBMCs were washed with RPMI (10\% FCS) and adjusted in the same medium to the desired $\mathrm{E} / \mathrm{T}$ ratio, and bsAb dilution to the required concentrations was carried out using PBS/0.1\% human serum albumin. The cytotoxic reaction was performed by mixing $0.3 \times 10^{5}$ calcein-AM-labeled target cells with $0.3 \times 10^{6} \mathrm{~T}$ cells or PBMCs (E/T ratio of 10:1) in $5 \% \mathrm{CO}_{2}$ at $37^{\circ} \mathrm{C}$, followed by the addition of $20 \mu \mathrm{l}$ of antibody (200 $\mu 1$ of total volume). Cell lysis was then assessed after 4 hours. The released fluorescent dye into the supernatant was quantified by synergy4 fluorescence reader (Germany). The fluorescence signal was measured from cells lacking cytotoxic compound, while the bsAb was used as a negative control. For positive control NALM-6 cells (without bscCD19 $\times$ CD3) were nonspecifically lyzed in $1 \%$ saponin for 10 minutes (20 $\mu \mathrm{l}$ of final volume). Specific cytotoxicity was calculated based on the following formula:

$\mathrm{F}$ (sample) $-\mathrm{F}$ (control)/[F(total lysis) $-\mathrm{F}($ control $)] \times 100$

\section{Ethical statement}

The above-mentioned sampling protocols were approved by the Research Ethics Committee of Pasteur Institute of Tehran, Iran (ethical code:
IR.PII.REC.1394.38). Written informed consents were provided by all the participants.

\section{RESULTS}

\section{Expression vector construction}

The encoding sequence of blinatumomab (a BiTE antibody) was cloned into the multiple cloning site of pcDNA3.1 (+) vector using NheI and HindIII restriction enzymes to produce the first construct, pcDNABi. Cloning confirmation was performed by double digestion using the two mentioned enzymes, showing the excision of a 1600-bp insert (Fig. 1A). For making the second construct, pcDNABiW, the DNA sequences of antibody and WPRE element were successfully cloned into the pcDNA3.1 (+) vector using NheI and EcoRI restriction enzymes, and then cloning was confirmed by EcoRV digestion of the final construct (Fig. 1B). The fidelity of cloning for both constructs was ultimately confirmed by sequencing.

\section{Expression analysis}

Following transient transfection of Expi293F $\mathrm{F}^{\mathrm{TM}}$ cells, the expressed bsAb was purified from the supernatant of the cells, bearing a yield of $2.3 \mathrm{mg} / \mathrm{L}$. For the transient expression of recombinant antibody in Expi293F $\mathrm{F}^{\mathrm{TM}}$ cells, a mixture of pcDNABi and pb513b1 or a mixture of pcDNABiW and pb513b1 was independently co-transfected into the cells. The pcDNABi or pcDNABiW ratio to pb513b1 was $1: 10$ in each experiment. On day seven post transfection, the expressed bsAb was collected from the supernatant of each set of transfected cells independently, followed by elution under identical conditions (using the same column, sample volume, and elution condition) for
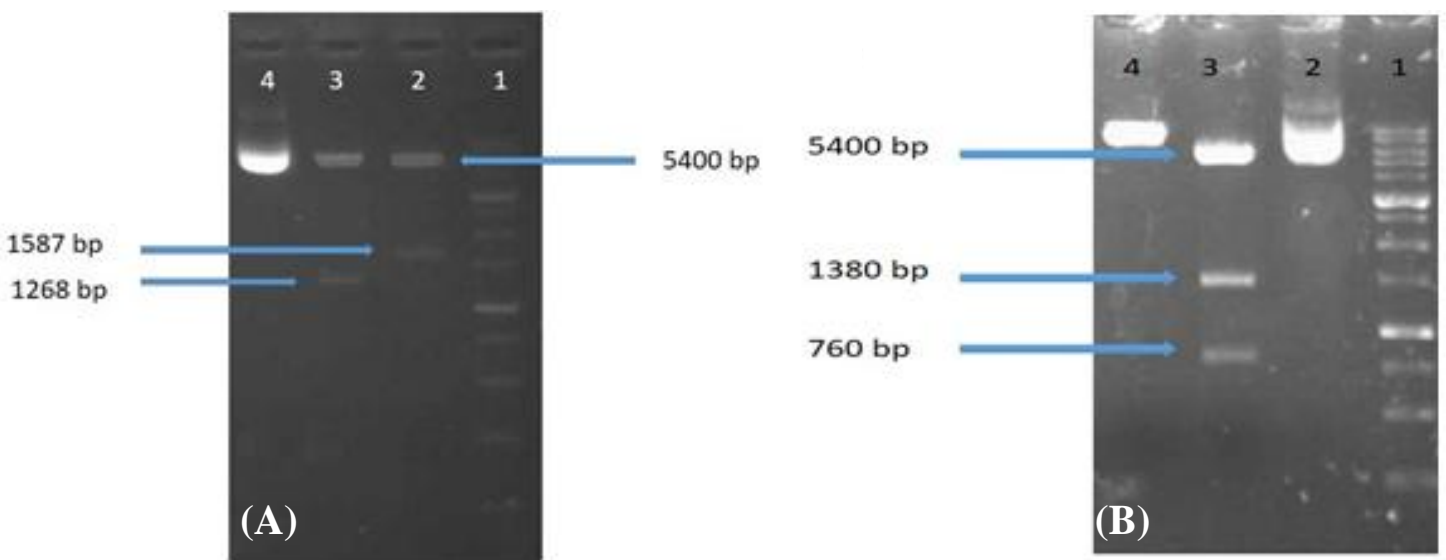

Fig. 1. Cloning confirmation of pcDNABi (A) and pcDNABiW (B). (A) Lane 1, 1-kbp DNA marker; lane 2, digested pcDNABi vector by NheI and HindIII restriction enzymes; lane 3, XhoI digested pcDNABi vector; lane 4, undigested pcDNABi vector; (B) Lane 1, 1-kbp DNA marker; lane 2, undigested pcDNA3.1 vector; lane 3, digestion of pcDNABiW vector with EcoRV generating three fragments: 5400, 1400, and $760 \mathrm{bp}$; lane 4, digested the pcDNABiW vector with NheI restriction enzyme. 


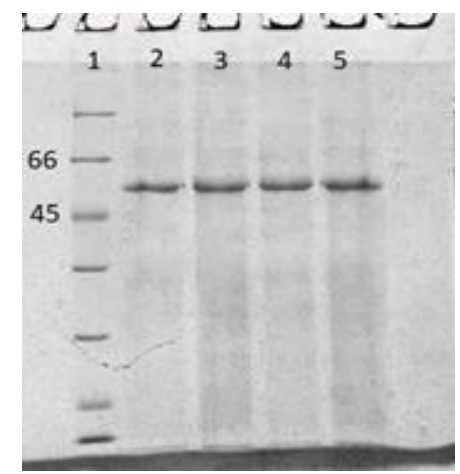

Fig. 2. SDS-PAGE analysis of a purified bscCD19 $\times$ CD3 . Lane 1, protein ladder $116-14 \mathrm{kDa}$; lanes $2-5$, the eluted fraction of bsAb.

comparison (Fig. 2). The eluted fraction was measured using a NanoDrop spectrophotometer (Thermo Fisher Scientific, USA). Interestingly, the production of bsAb showed a 1.8-fold increase in WPRE plus construct (pcDNABiW) compared with antibodies expressed and purified from WPRE minus construct (pcDNABi). Spike-in control using GFP-expressing reporter plasmid (pb513b1) was applied for the normalization of sample-to-sample variations in transfection. Assessment by flow cytometry showed negligible differences between the two experiments, i.e. transfection of pcDNABi and pb513b1 or transfection of pcDNABiW and pb513b1 (Fig. 3). The eluted protein was detected by anti-polyhistidine antibody conjugated to HRP demonstrated by Western blot analysis. The molecular weight of the purified protein was detected to be around $55 \mathrm{kDa}$ (Fig. 4).

\section{Binding properties}

Binding properties of the expressed bsAb bscCD19 $\times$ CD3 was evaluated by flow cytometry. To assess the binding properties of the anti-CD3 moiety, we used

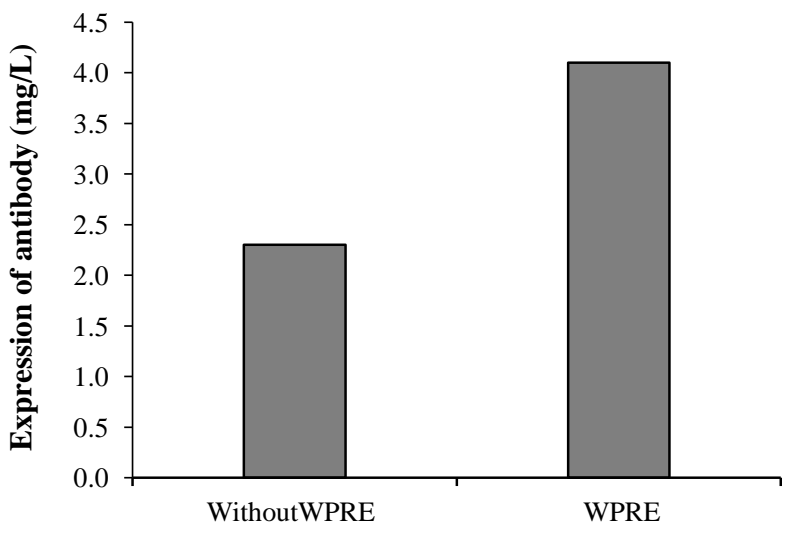

Fig. 3. The mAb expression level in Expi293FTM cells in the presence and absence of WPRE element. WPRE enhanced the expression level of bscCD19 $\times$ CD3 . two cell types, Jurkat cells as a $\mathrm{CD}^{+}$cell line and PBMC (isolated from healthy human peripheral blood containing $\mathrm{CD}^{+} \mathrm{T}$ lymphocytes). Both cell types were incubated with the purified bscCD19 $\times$ CD3 antibody $(200 \mu \mathrm{g} / \mathrm{mL})$ and then labeled with the anti-His-tagPerCP as the secondary antibody (see details in Materials and Methods). As shown in Figure 5, attachment of the $\mathrm{bscCD} 19 \times \mathrm{CD} 3$ to the $\mathrm{CD} 3$ receptor was observed in both Jurkat $\mathrm{CD}^{+}$cell line and PBMC.

The $\mathrm{CD} 19^{+}$B-cell line (NALM-6) was used to evaluate the binding properties of the CD19 moiety. For this purpose, NALM-6 cells incubated with the primary bsAb $($ bscCD $19 \times \mathrm{CD} 3)$ were mixed with anti-

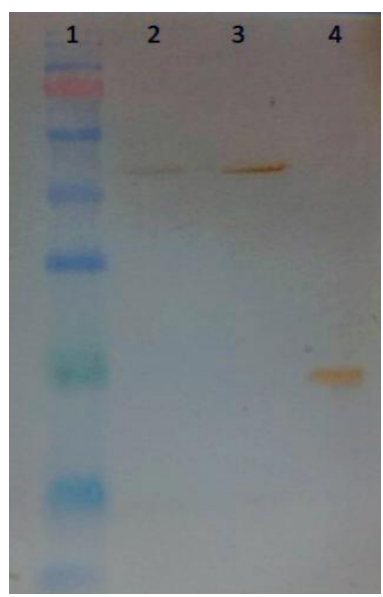

Fig. 4. Western blot analysis of bsAb with HRP-conjugated anti-polyhistidine antibody. Lane 1, protein ladder (10-180 $\mathrm{kDa}$ ); lanes 2 and 3, eluted supernatant of transfected Expi293F ${ }^{\mathrm{TM}}$ cells; lane 4, His-tag positive control.

His-tag-PerCP, a PerCP-labeled anti-His IgG antibody (as mentioned in Materials and Methods), followed by FACS analysis that showed acceptable binding properties. Interestingly, no binding was detected on the $\mathrm{CD} 19$, the $\mathrm{CD} 3$ double-negative $\mathrm{CHO}$ cell line (Fig. 5).

\section{Bioactivity assay}

The fluorochrome calcein- $\mathrm{AM}^{33}$ was used as a fluorescence-based cytotoxicity assay, which is trapped inside living cells and released only upon cell lysis, resulting in the appearance of the fluorescent dye in the supernatant. Calcein-AM-labeled NALM-6 B cells were mixed with $\mathrm{T}$ cells isolated from PBMCs at a 10:1 ratio. After four hours of incubation, calcein-AM was determined in the cell culture medium (sup) by a fluorometer. Figure 5 indicates the dose-response curves of five donor T-cell preparations. A variable percentage of cell lysis and different $\mathrm{ED}_{50}$ values were obtained with effector cells from five donors. The 


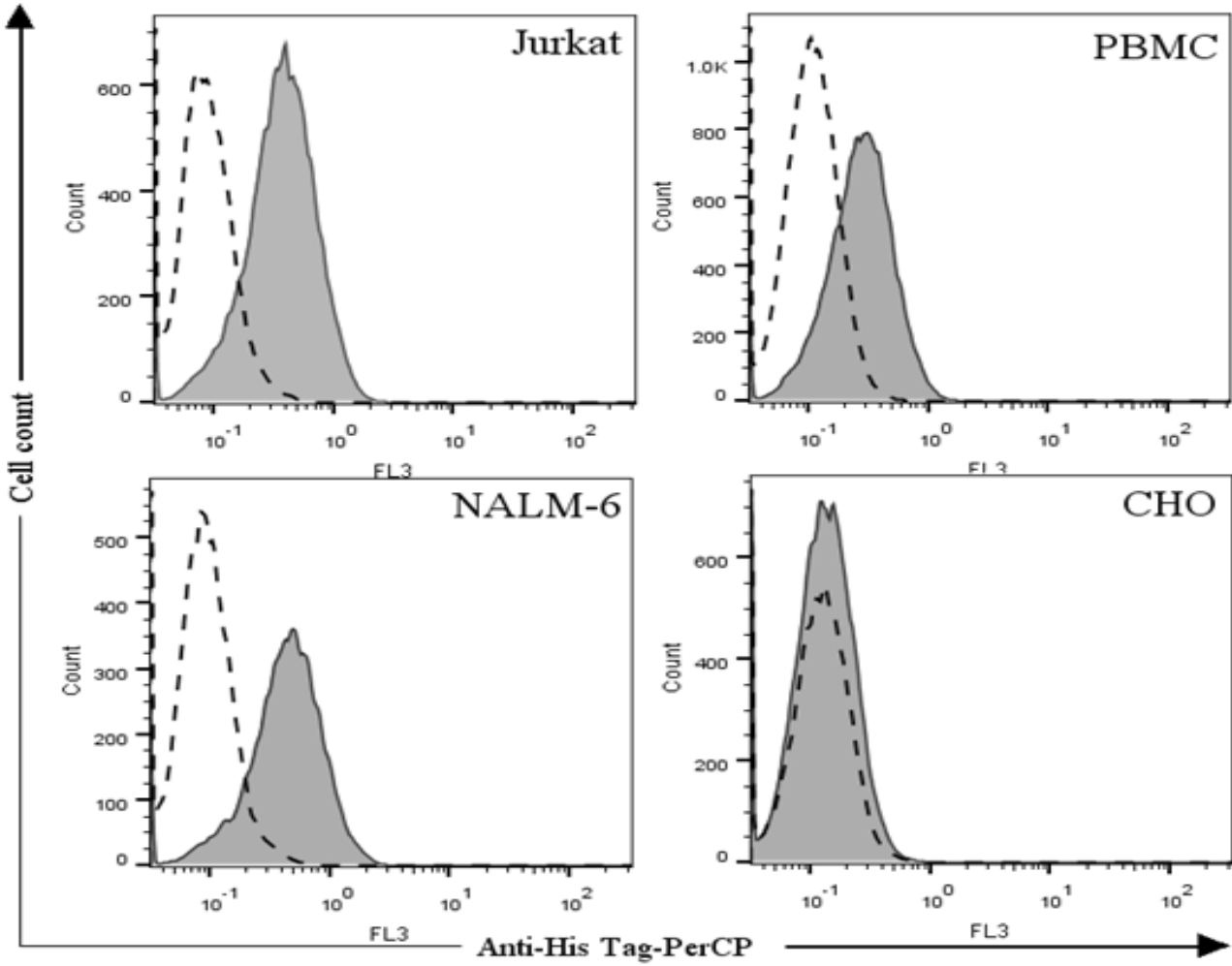

Fig. 5. Binding properties of the bscCD $19 \times \mathrm{CD} 3$. Binding specifications were evaluated by flow cytometry. The Jurkat cell line and PMBCs from healthy donor were used to assess the anti-CD3 binding potency of the bscCD19 $\times$ CD3, while CD19-positive B-cell line (NALM-6) were used to evaluate binding properties of the CD19 end of the bsAb. Anti-His-tag PerCP conjugate was employed as a secondary antibody. As shown in the Figure, both ends of bscCD19 $\times$ CD3 reveled significant binding to their receptor. $\mathrm{CHO}^{\left(\mathrm{CD}^{-}\right.}$ $\mathrm{CD}^{-}$) cells were applied as a negative control, showing no binding of the bscCD19 $\times$CD3 to this cell. Dotted lines represent cells untreated with bsAb.

value and percentage of $\mathrm{ED}_{50}$ and cell lysis ranged from $52 \mathrm{pg} / \mathrm{ml}$ to $109 \mathrm{pg} / \mathrm{ml}$ and $26 \%$ to $62 \%$, respectively. Notably, we observed that the $\mathrm{ED}_{50}$ value was inversely proportional to the cell lysis, i.e. the higher the percentage of cell lysis, the lower the ED value. However, the correlation was not completely linear (Fig. 6). In four out of the five cases, bs Ab was effective in concentrations below $100 \mathrm{pg} / \mathrm{ml}$, and the mean of $\mathrm{ED}_{50}$ value for these five donors was $78 \mathrm{pg} / \mathrm{ml}$ (Fig. 6). To measure the targeting efficiency of bsAb at different $\mathrm{E} / \mathrm{T}$ ratios, the $\mathrm{mAb}$ was examined at several $\mathrm{E} / \mathrm{T}$ ratios $(10: 1,5: 1$, and 2.5:1) by using NALM-6 and T cells as target and effector cells, respectively. As depicted in Figure 7, the $\mathrm{ED}_{50}$ values for these ratios were 52,61 , and $65 \mathrm{pg} / \mathrm{ml}$, respectively. This observation demonstrated that the $\mathrm{ED}_{50}$ values slightly increased in response to the decrease in $\mathrm{E} / \mathrm{T}$ ratios with close cell lysis ratio values of 10:1 and 5:1 (63\% vs. $56 \%$, respectively). Our findings proved the effectiveness of anti CD3 $\times$ CD19 bsAb at low E/T ratios. Moreover, in contrast to some other CD19targeting bispecific antibodies, a low $\mathrm{E} / \mathrm{T}$ ratio is enough for cell lysis.

\section{DISCUSSION}

Mammalian expression systems are important, particularly in the production of biopharmaceuticals. In this context, transient gene expression is a primary step that can cost-effectively produce quite enough amounts of target proteins in a short time for preclinical studies. HEK293 cell line has been used for decades as a cell line of choice for transient gene expression, due to its high transfection efficiency as well as high protein production yield. However, this cell line is adherent, limiting its scale-up and expansion. In contrast, Expi293, a derivative of HEK293, is a suspension cell line with the capability of reaching high cell densities.

Vector engineering, the same as cell line engineering and process development, is a fundamental approach for enhancing the production yield of recombinant proteins. PTREs have been frequently used to engineer expression vectors, among which WPRE insertion considerably increases the expression level of transgenes in some hosts ${ }^{[27,34]}$.

In the current study, we expressed and purified blinatumomab and measured its cytotoxicity and 


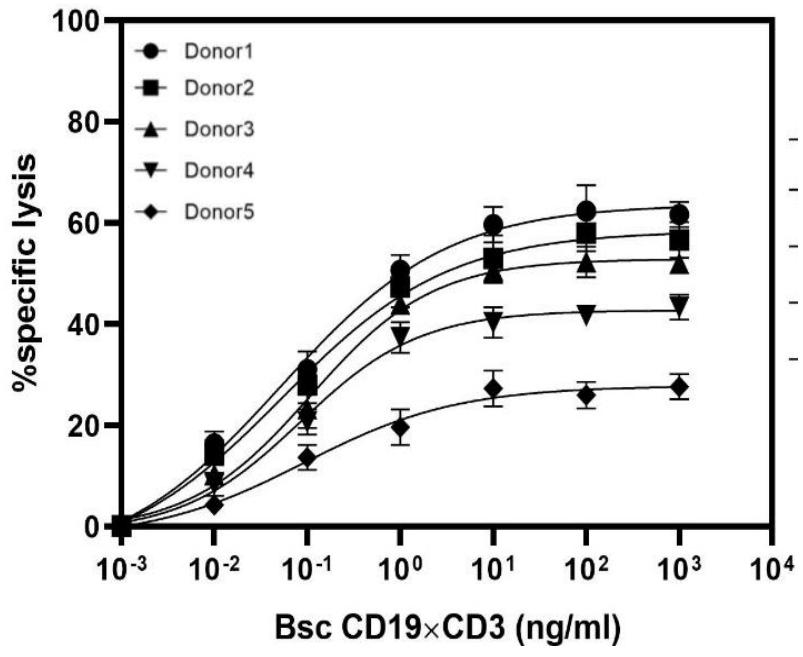

Fig. 6. Dose-response analysis of peripheral $\mathrm{T}$ cell of five healthy donors (effector cells) and NALM-6 cells (target cells). Calcein-AM release assay was performed at 10:1 ratio after four hours. Error bars give mean ( \pm standard deviation) values of triplicated measurement.

binding properties in a mammalian expression system and evaluated the effect of the WPRE sequence on the expression levels of the mAb. The bsAb was expressed from a pcDNA3.1 expression vector under the strong Cytomegalovirus promoter activity. Our analysis showed about two-fold increase in the expression level of bispecific antibody when WPRE was present ( $4.1 \mathrm{mg} / \mathrm{L}$ from pcDNABiW vector) compared with the one lacking it $(2.3 \mathrm{mg} / \mathrm{L}$ from pcDNABi vector). This result is consistent with previous studies that demonstrated WPRE can enhance the expression levels of recombinant proteins. Kim et al. ${ }^{[31]}$ reported that WPRE increases the expression levels of a full-length

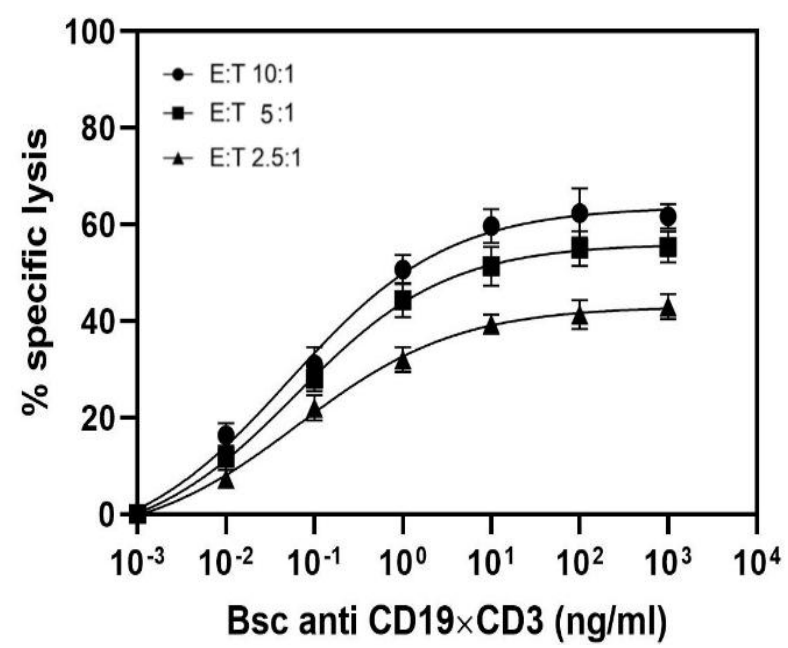

Fig. 7. The effect of different E/T ratios on cell lysis activity of bscCD19 $\times$ CD3 . Dose-response analysis is based on calceinAM cytotoxicity assay. Error bars give mean $( \pm$ standard deviation) values of triplicated measurement. antibody from $19.3 \mathrm{mg} / \mathrm{L}$ to $40.5 \mathrm{mg} / \mathrm{L}$, bringing the enhancement to about 2.1-fold in HEK293E cells. Ho et al. ${ }^{[27]}$ showed WPRE can promote the expression of luciferase by 2.6-fold in HEK293 cells, but had no positive effects in $\mathrm{CHO} \mathrm{K} 1$ cells. Hlavaty et al. ${ }^{[35]}$ also reported that WPRE enhanced recombinant protein expression level up to three folds in HEK293 cell line, and a combination of WPRE with other PTREs has been shown to increase the expression levels up to 10.5 folds in HEK cells. Besides these positive results, there are also some reports showing no significant enhancements in the expression levels of transgenes by WPRE $^{[27]}$. This discrepancy can be interpreted as context-dependent effects ${ }^{[36]}$ or promoter and cell linespecificity and activity ${ }^{[37]}$ of WPRE. Therefore, particularly for industrial applications, it is important to evaluate the impact of WPRE insertion individually on each specific construct, candidate gene, or host cell line.

In the present investigation, we demonstrated that the use of WPRE in the pcDNA3.1 expression vector for the expression of bscCD19 $\times$ CD3 antibody in Expi293FTM cells effectively increase the expression levels of the transgene. Previous studies have exhibited that bscCD19 $\times \mathrm{CD} 3$ is effective at low $\mathrm{pg} / \mathrm{ml}$ ranges and low $\mathrm{E} / \mathrm{T}$ ratios ${ }^{[38,39]}$. Consistent with those reports, functional analysis displayed that the produced antibody using our expression system was capable of lysing target cells at sub $\mathrm{ng} / \mathrm{ml}$ concentrations and low $\mathrm{E} / \mathrm{T}$ ratios. In contrast with other bispecific technologies, such as quadroma or diabody requiring much higher concentrations (i.e. 100 nanograms to micrograms of antibodies), the BiTE system induces cell lysis at sub $\mathrm{ng} / \mathrm{ml}$ concentrations, in a short period of time at low E/T ratios ${ }^{[40]}$. Additionally, using the Tcell activation of BiTE system is self-sufficient and independent of extra stimuli such as IL-2 and gamma interferon, a requirement for other similar systems ${ }^{[38]}$.

In this study, we demonstrated the successful production and purification of a bsAb; bscCD19 $\times$ CD3, in Expi293 suspension cells via the insertion of a WPRE sequence in the expression cassette. The produced antibody showed full functionality at $\mathrm{pg} / \mathrm{ml}$ concentrations. Our new expression system could be proposed for the production of this antibody at an industrial scale.

\section{ACKNOWLEDGEMENTS}

This study was supported by a Ph.D. student grant (No. 93/0110/651) from the Pasteur Institute of Iran, Tehran.

CONFLICT OF INTEREST. None declared. 


\section{REFERENCES}

1. Goebeler ME, Bargou R. Blinatumomab: a CD19/CD3 bispecific $\mathrm{T}$ cell engager (BiTE) with unique anti-tumor efficacy. Leukemia and lymphoma 2016; 57(5): 10211032.

2. Ventola CL. Cancer immunotherapy, part 1: Current strategies and agents. Pharmacy and therapeutics 2017; 42(6): 375-383.

3. Scott AM, Wolchok JD, Old LJ. Antibody therapy of cancer. Nature reviews cancer 2012; 12: 278-287.

4. Chames P, Van Regenmortel M, Weiss E, Baty D. Therapeutic antibodies: Successes, limitations and hopes for the future. British journal of pharmacology 2009; 157(2): 220-233.

5. Beckman RA, Weiner LM, Davis HM. Antibody constructs in cancer therapy: protein engineering strategies to improve exposure in solid tumors. Cancer 2007; 109(2): 170-179.

6. Cruz E, Kayser V. Monoclonal antibody therapy of solid tumors: Clinical limitations and novel strategies to enhance treatment efficacy. Biologics: targets and therapy 2019; 13: 33-51.

7. Nunez-Prado N, Compte $M$, Harwood S, ÁlvarezMéndez A, Lykkemark S, Sanz L, Álvarez-Vallina L. The coming of age of engineered multivalent antibodies. Drug discovery today 2015; 20(5): 588-594.

8. Krishnamurthy A, Jimeno A. Bispecific antibodies for cancer therapy: A review. Pharmacology and therapeutics 2018; 185: 122-134.

9. May C, Sapra P, Gerber HP. Advances in bispecific biotherapeutics for the treatment of cancer. Biochemical pharmacology 2012; 84(9): 1105-1112.

10. Thakur A, Lum LG. "NextGen" biologics: Bispecific antibodies and emerging clinical results. Expert opinion on biological therapy 2016; 16(5): 675-688.

11. Husain B, Ellerman D. Expanding the boundaries of biotherapeutics with bispecific antibodies. Biodrugs 2018; 32(5): 441-464.

12. Fan D, Li W, Yang Y, Zhang X, Zhang Q, Yan Y, Yang $\mathrm{M}$, Wang J, Xiong D. Redirection of CD4+ and CD8+ T lymphocytes via an anti-CD3x anti-CD19 bi-specific antibody combined with cytosine arabinoside and the efficient lysis of patient-derived B-ALL cells. Journal of hematology and oncology 2015; 8: 108.

13. Ribera JM. Efficacy and safety of bispecific T-cell engager blinatumomab and the potential to improve leukemia-free survival in B-cell acute lymphoblastic leukemia. Expert review of hematology 2017; 10(12): 1057-1067.

14. Brandl C, Haas C, d'Argouges S, Fisch T, Kufer P, Brischwein K, Prang N, Bargou R, Suzich J, Baeuerle PA, Hofmeister R. The effect of dexamethasone on polyclonal $\mathrm{T}$ cell activation and redirected target cell lysis as induced by a CD19/CD3-bispecific singlechain antibody construct. Cancer immunology, immunotherapy 2007; 56(10): 1551-1563.

15. Hoffman L, Gore L. Blinatumomab, a bi-specific antiCD19/CD3 BiTE® antibody for the treatment of acute lymphoblastic leukemia: Perspectives and current pediatric applications. Frontiers in oncology 2014; 4: 63.

16. Nagorsen D, Baeuerle PA. Immunomodulatory therapy of cancer with $\mathrm{T}$ cell-engaging BiTE antibody blinatumomab. Experimental cell research 2011; 317(9): 1255-1260.

17. May MB, Glode A. Blinatumomab: A Novel, Bispecific, T-cell Engaging Antibody. Oxford University Press, 2016.

18. Portell CA, Wenzell CM, Advani AS. Clinical and pharmacologic aspects of blinatumomab in the treatment of B-cell acute lymphoblastic leukemia. Clinical pharmacology: advances and applications 2013; 5 (Suppl 1): 5-11.

19. Buie LW, Pecoraro JJ, Horvat TZ, Daley RJ. Blinatumomab: a first-in-class bispecific T-cell engager for precursor B-cell acute lymphoblastic leukemia. Annals of pharmacotherapy 2015; 49(9): 1057-1067.

20. Kuo S-R, Wong L, Liu J-S. Engineering a CD123xCD3 bispecific $\mathrm{scFv}$ immunofusion for the treatment of leukemia and elimination of leukemia stem cells. Protein engineering, design and selection 2012; 25(10): 561-569.

21. Wang Q, Chen Y, Park J, Liu X, Hu Y, Wang T, McFarland K, Betenbaugh MJ. Design and production of bispecific antibodies. Antibodies 2019; 8(3): 43.

22. Regula JT, von Leithner PL, Foxton R, Barathi VA, Cheung CM, Bo Tun SB, Wey YS, Iwata D, Dostalek M, Moelleken J, Stubenrauch KG, Nogoceke E, Widmer G, Strassburger P, Koss MJ, Klein C, Shima DT, Hartmann G. Targeting key angiogenic pathways with a bispecific CrossMAb optimized for neovascular eye diseases. EMBO molecular medicine 2016; 8(11): 12651288.

23. Huang Y, Yu J, Lanzi A, Yao X, Andrews CD, Tsai L, Gajjar MR, Sun M, Seaman MS, Padte NN, Ho DD. Engineered bispecific antibodies with exquisite HIV-1neutralizing activity. Cell 2016; 165(7): 1621-1631.

24. Baldi L, Hacker DL, Adam M, Wurm FM. Recombinant protein production by large-scale transient gene expression in mammalian cells: State of the art and future perspectives. Biotechnology letters 2007; 29(5): 677-684.

25. Wurm F, Bernard A. Large-scale transient expression in mammalian cells for recombinant protein production. Current opinion in biotechnology 1999; 10(2): 156-159.

26. Matasci M, Hacker DL, Baldi L, Wurm FM. Recombinant therapeutic protein production in cultivated mammalian cells: current status and future prospects. Drug discovery today: technologies 2008; 5(2-3): e37-e42.

27. Mariati, Ho SC, Yap MG, Yang Y. Evaluating posttranscriptional regulatory elements for enhancing transient gene expression levels in $\mathrm{CHO} \mathrm{K} 1$ and HEK293 cells. Protein expression and purification 2010; 69(1): 9-15.

28. Brun S, Faucon-Biguet N, Mallet J. Optimization of transgene expression at the posttranscriptional level in neural cells: implications for gene therapy. Molecular therapy $2003 ; 7(6)$ : 782-789. 
29. $\mathrm{Xu}$ ZL, Mizuguchi H, Mayumi T, Hayakawa $T$. Woodchuck hepatitis virus post-transcriptional regulation element enhances transgene expression from adenovirus vectors. Biochimica et biophysica acta 2003; 1621(3): 266-271.

30. Zufferey R, Donello JE, Trono D, Hope TJ. Woodchuck hepatitis virus posttranscriptional regulatory element enhances expression of transgenes delivered by retroviral vectors. Journal of virology 1999; 73(4): 2886-2892.

31. Kim KS, Kim MS, Moon JH, Jeong MS, Kim J, Lee GM, Myung PK. Enhancement of recombinant antibody production in HEK 293E cells by WPRE. Biotechnology and bioprocess engineering 2009; 14: 633.

32. Kokkinopoulos D, Perez S, Sotiriadou R, Stinios J, Papamichail M. The use of nylon wool for the isolation of $\mathrm{T}$ lymphocyte subpopulations. Journal of immunological methods 1992; 154(1): 1-6.

33. Wang XM, Terasaki PI, Rankin Jr GW, Chia D, Zhong HP, Hardy S. A new microcellular cytotoxicity test based on calcein AM release. Human immunology 1993; 37(4): 264-270.

34. Loeb JE, Cordier WS, Harris ME, Weitzman MD, Hope TJ. Enhanced expression of transgenes from adenoassociated virus vectors with the woodchuck hepatitis virus posttranscriptional regulatory element: Implications for gene therapy. Human gene therapy 1999; 10(14): 2295-2305.

35. Hlavaty J, Schittmayer M, Stracke A, Jandl G, Knapp E, Felber BK, Salmons B, Günzburg WH, Renner M.
Effect of posttranscriptional regulatory elements on transgene expression and virus production in the context of retrovirus vectors. Virology 2005; 341(1): 1-11.

36. Schambach A, Wodrich $\mathrm{H}$, Hildinger $\mathrm{M}$, Bohne J, Kräusslich HG, Baum C. Context dependence of different modules for posttranscriptional enhancement of gene expression from retroviral vectors. Molecular therapy 2000; 2(5): 435-445.

37. Klein R, Ruttkowski B, Knapp E, Salmons B, Günzburg WH, Hohenadl C. WPRE-mediated enhancement of gene expression is promoter and cell line specific. Gene 2006; 372: 153-161.

38. Dreier T, Lorenczewski G, Brandl C, Hoffmann P, Syring U, Hanakam F, Kufer P, Riethmuller G, Bargou R, Baeuerle PA. Extremely potent, rapid and costimulation-independent cytotoxic T-cell response against lymphoma cells catalyzed by a single-chain bispecific antibody. International journal of cancer 2002; 100(6): 690-697.

39. Hoffmann P, Hofmeister R, Brischwein K, Brandl C, Crommer S, Bargou R, Itin C, Prang N, Baeuerle PA. Serial killing of tumor cells by cytotoxic $\mathrm{T}$ cells redirected with a CD19-/CD3-bispecific single-chain antibody construct. International journal of cancer 2005; 115(1): 98-104.

40. Gruber M, Schodin BA, Wilson ER, Kranz DM. Efficient tumor cell lysis mediated by a bispecific single chain antibody expressed in Escherichia coli. The journal of immunology 1994; 152(11): 5368-5374. 